\title{
Miranda
}

Revue pluridisciplinaire du monde anglophone /

Multidisciplinary peer-reviewed journal on the English-

speaking world

$12 \mid 2016$

Mapping gender. Old images ; new figures

\section{Glissement du mode « majeur » au mode « mineur » dans The Best of Simple de Langston Hughes}

\section{Christine Dualé}

\section{OpenEdition}

Journals

Édition électronique

URL : http://journals.openedition.org/miranda/8102

DOI : $10.4000 /$ miranda. 8102

ISSN : 2108-6559

Éditeur

Université Toulouse - Jean Jaurès

\section{Référence électronique}

Christine Dualé, «Glissement du mode « majeur » au mode « mineur » dans The Best of Simple de Langston Hughes », Miranda [En ligne], 12 | 2016, mis en ligne le 26 février 2016, consulté le 16 février 2021. URL : http://journals.openedition.org/miranda/8102 ; DOI : https://doi.org/10.4000/miranda. 8102

Ce document a été généré automatiquement le 16 février 2021.

\section{(c) $(1)$}

Miranda is licensed under a Creative Commons Attribution-NonCommercial-NoDerivatives 4.0 International License. 


\title{
Glissement du mode « majeur » au mode " mineur » dans The Best of Simple de Langston Hughes
}

\author{
Christine Dualé
}

1 Au cours de sa carrière artistique, Langston Hughes a maintes fois exprimé le désir de retranscrire l'âme et les émotions de la communauté noire à travers son écriture. Grâce à sa conception personnelle d'une "esthétique noire » Hughes contribua aux riches heures de la Renaissance de Harlem en revendiquant une autre image des Noirs et tout en revalorisant des aspects occultés de l'héritage culturel noir américain. Le blues occupa une place prépondérante dans la vie et l'écriture de l'écrivain pour qui la recherche d'une appartenance remontant aux origines africaines fut un point d'ancrage essentiel. Il expérimenta aussi d'autres formes d'écriture et en s'éloignant de la tradition culturelle anglo-saxonne et européenne.

Influencé par "l'école de Harlem », mais conscient que sa création devait passer par une écriture différente, Hughes fut le garant d'un nouveau style en construisant sa propre écriture sur des principes fédérateurs et des stratégies communes. A travers une " écriture métissée ${ }^{1}$ », Hughes réussit à s'approprier les modes d'expression dominants et à les restituer avec les outils de la culture noire américaine. Hughes proposa une écriture sans précédent et « révolutionnaire » au sens deleuzien.

3 Après avoir exploré différentes formes poétiques, Hughes créa Simple, un personnage fictif de nouvelles écrites sur une période de vingt-trois ans ${ }^{2}$, qui devint le personnage phare de son œuvre et qui cristallise les différentes «modulations » et «minorations » de son écriture. Intimement lié à l'ère du blues et du jazz, Simple évolue pendant la Renaissance de Harlem (1924-1930) et s'exprime dans une langue qui s'éloigne de l'anglais standard ce qui lui donne l'occasion, tout au long de ses péripéties, de dévoiler l'âme et l'expérience noires. Dans The Best of Simple (L'Ingénu de Harlem en français), la présence de doubles, c'est-à-dire de doubles-entendre, de doubles sens, de doubles jeux apparaît comme une volonté de jouer avec le lecteur et de brouiller les pistes. Qui parle? Et que souhaitait révéler l'auteur? Comment la langue est-elle 
« redistribuée ${ }^{3} »$ (Barthes 13)? Hughes joue avec ces techniques qui sont pour lui autant de masques qui lui permettent de prêter différentes voix à Simple et de retranscrire au plus près la vie des Noirs les plus humbles.

4 Nous allons voir comment, à travers des chemins détournés, l'écriture de The Best of Simple présente des «strates » successives et se structure autour d'assemblages et de réflexions en perpétuels mouvements. Pour ce faire, nous nous appuierons sur les concepts théorisés par Gilles Deleuze et Félix Guattari dans Mille Plateaux. Telles des strates, la langue de Hughes est faite d'unité et de diversité et doit s'appréhender dans sa multiplicité et sa variabilité :

Dans une strate il y a des doubles-pinces partout, des double binds, des homards partout, dans toutes les directions, une multiplicité d'articulations doubles qui traversent tantôt l'expression, tantôt le contenu (Deleuze, Guattari MP 60).

Utiliser l'approche deleuzienne pour évoquer l'écriture de Hughes peut paraitre comme n'allant pas de soi. Pourtant, à y bien regarder, l'écriture de Hughes se démultiplie, connecte des rhizomes ${ }^{4}$, renvoie à la fois à la mémoire collective noire, et à l'héritage noir, mais aussi à l'héritage de la tradition littéraire anglo-saxonne et européenne pour déterritorialiser ${ }^{5}$ et re-territorialiser des modes d'expression sans cesse modelés et remodelés.

\section{La « marge » et le « mineur » dans l'écriture de Langston Hughes}

6 L'esthétique de Hughes à travers l'écriture de l'oralité évoque la langue mineure deleuzienne. Dans The Best of Simple, l'originalité de Hughes est de proposer un texte très métissé, empruntant ses formes à la fois au folklore africain et noir américain sans jamais s'éloigner de la tradition américaine et européenne. Synthèse "rizhomatique » de la tradition littéraire, poétique et langagière africaine et anglo-saxonne, Hughes propose un style nouveau où son personnage central est constamment tiraillé entre deux cultures, deux histoires, deux expériences, et où l'écriture est travaillée afin de proposer des variations langagières. La langue est déterritorialisée pour, sous d'autres modalités, être re-territorialisée. En ce sens, le travail de Hughes dans The Best of Simple est une parfaite illustration de la « littérature mineure » telle qu'elle a été théorisée par Gilles Deleuze :

(...) La littérature présente déjà deux aspects, dans la mesure où elle opère une décomposition ou une destruction de la langue maternelle, mais aussi l'invention d'une nouvelle langue dans la langue, par création de syntaxe (Deleuze, CC 16).

7 Outre le travail sur la langue, le texte de Hughes renvoie constamment à certains aspects du folklore noir, notamment à travers l'écriture de l'oralité, tout en empruntant à la tradition littéraire sur fond de critique plus ou moins acerbe de la société américaine du début du vingtième siècle. L'extrait suivant est un exemple du travail de mosaïque que représentent ces tranches de vie dans leur ensemble :

Then, if I was an artist, I would put all that into a comic book. I wonder why somebody don't make comic books out of the funny way white folks in America behave - talking democracy out of one side of their mouth and, "Negro, stay in your place", out of the other. I wish I could draw, I would make me such a book. I would start a whole series of comics which I bet would sell a million copies - Jess Simple's Jim Crow Jive, would be the title. I would make my books in both English and Spanish so the Puerto Ricans could laugh, too (Puerto-Ricains, 218). ${ }^{6}$ 
Le glissement de l'auteur de la marge à la norme, cette ligne de fuite, pour reprendre Deleuze, illustre à quel point le travail d'écriture de Hughes est caractéristique de la littérature « mineure » noire :

Une littérature mineure n'est pas celle d'une langue mineure, plutôt celle qu'une minorité fait dans une langue majeure. (...) Elle fait subir à une langue dominante un traitement qui la rend étrangère à elle-même et la fait tendre vers ses extrêmes ou ses limites (Deleuze, Guattari, KLM 29, 42).

En n'écrivant plus uniquement pour un lectorat blanc, Hughes explore de nouvelles formes d'écriture pour s'éloigner des portraits stéréotypés et plein de bons sentiments de la littérature blanche. L'artiste trouve la voie d'une expression libre et nouvelle.

Sa constante préoccupation fut de restituer au plus près l'authenticité de la voix noire. Alors que ses contemporains prirent leur distance avec la culture populaire, Hughes privilégia la culture noire populaire dans l'élaboration de son écriture tout en empruntant à la fois au folklore noir et à la tradition littéraire classique. Dans Apple Strudel (114-116) en citant Walden de Henry Thoreau ${ }^{7}$, Hughes fait explicitement allusion à la tradition littéraire à travers son propre personnage. En effet, comme le personnage principal de Walden, dont l'isolement dans la nature est favorable à l'introspection, Simple fait part de ses observations sur la condition noire dans un monde en pleine mutation. Cette référence n'est pas non plus un hasard si l'on pense à l'engagement abolitionniste de Thoreau qui prit, en son temps, des positions radicales contre l'esclavage. D'autres allusions subtiles à la tradition littéraire émaillent le récit, notamment lorsque Boyd, le narrateur, récite un poème de Ben Jonson (1573-1637) dont Simple n'a jamais entendu parler :

Drink to me only with thine eyes,

And I will pledge with mine,

Or leave a kiss but in the cup,

And I'll not ask for wine.

( «Chanson à Célie », Ben Jonson", dans Cocktail Sip, 163).

11 Ce poème sert de faire-valoir à Boyd pour souligner l'ignorance de Simple et, au contraire, la bonne éducation du narrateur. Des allusions à la tradition européenne apparaissent d'ailleurs à plusieurs reprises: la nouvelle Cocktail Sip par exemple, s'ouvre sur l'échange suivant: «Boyd: You sound like an Elizabethan. What's up? / Lizzie who? asked Simple » (Cocktail Sip, 116).

Un autre genre littéraire que Hughes emprunte à la tradition est celui du «tall tale». Dans la tradition folklorique américaine le «tall tale» renvoie à des histoires incroyables et exagérées relatées de façon à faire croire en leur exactitude :

Le tall tale est " une galéjade qui vise à mettre à l'épreuve des étrangers naïfs prompts à idéaliser l'Ouest et à en faire un pays de légendes. Les personnages hauts en couleur qui défilent dans ces histoires hyperboliques sont décrits par des gentlemen, narrateurs occasionnels qui mettent à distance les personnages d'une nouvelle comédie humaine: rustauds, chasseurs fanfarons, ivrognes déchaînés, charlatans, colporteurs véreux, avocats marrons, médecins ambulants et spéculateurs fonciers (Royot, et.al 188).

Dans Feet Live Their Own Life (1-3), le récit hyperbolique qui ouvre The Best of Simple, Hughes revisite le genre :

"If you want to know about my life", said Simple, "don't look at my face, don't look at my hands. Look at my feet and see if you can tell how long I been standing on them". Can't you tell through the shoes I wear, that I been standing on these feet a long time and carrying some heavy burdens? (...) Everything I do is connected up 
with my past life. From Virginia to Joyce, from my wife to Zarita, from my mother's milk to this glass of beer, everything is connected up. (...) These feet have stood on every rock from the Rock of Ages to $135^{\text {th }}$ and Lenox. These feet have supported everything from a cotton bale to a hungry woman. These feet have walked ten thousand miles working for white folks and another ten thousand keeping up with colored. (...) If I just had four feet, I could have stood in more places longer. (...) If anybody was to write the history of my life, they should start with my feet" (Feet Live Their Own Life, 2-3). si le «tall tale » donna naissance à la culture de l'Ouest, Hughes, lui, donne naissance à une autre forme de culture noire, à mi-chemin entre tradition européenne et américaine mais aussi africaine, car l'emprunt à la tradition africaine est fondamental dans The Best of Simple. L'Afrique exotique, découverte par l'Amérique blanche et très en vogue à cette époque, fut exhumée par les intellectuels noirs en quête d'authenticité et à la recherche d'un style nouveau. En revisitant cette tradition, Hughes fut particulièrement innovant et montra sa volonté de remonter aux origines africaines par « refus d'allégeance aux valeurs de la culture dominante» (Fabre 90).

\section{« Devenir-révolutionnaire »}

Pour Deleuze, le « devenir-révolutionnaire » signifie : « connecter des hétérogènes », ce que Hughes propose dans son ouvrage en utilisant des stratégies textuelles et culturelles diverses et en se les appropriant. En s'intéressant à l'histoire africaine, les intellectuels noirs de la Renaissance ravivèrent et s'approprièrent des aspects de leur héritage racial occultés jusque-là et qui devinrent une véritable source d'inspiration :

Le Nègre nouveau éprouvait un besoin d'enracinement aux temps de la grande migration, lorsque les problèmes d'acculturation se posaient avec acuité. On le sent à la recherche d'une tradition, d'une appartenance qui remonte volontiers à l'esclavage et aux origines africaines (Fabre, 90).

Avec The Best of Simple, Hughes revisita un patrimoine remontant à l'esclavage comme pour mieux mettre à jour l'authenticité africaine de Simple.

Dans les sociétés d'Afrique noire la parole est un support culturel essentiel, au point que les ethnologues parlent de littérature orale africaine, qui assure la transmission de l'histoire entre les générations. Comme l'explique Vincent Hecquet :

La littérature orale est par nature un phénomène social, qui s'intègre dans le cadre des normes de sa prestation ou de la communication interpersonnelle. Les épopées sont relatées lors de réjouissances populaires ou de faits sociaux essentiels pour le groupe, par exemple l'initiation des jeunes. La musique accompagne le récit et participe à la construction du sens (Hecquet).

Dans le folklore africain, les contes relatent soit des événements imaginaires, soit la réalité et offrent un miroir de la société. Le conte a une valeur didactique car il doit susciter une prise de conscience chez ceux qui l'écoutent ${ }^{9}$. Réunis le soir autour de l'arbre à palabres, hommes et femmes écoutent le sage faire le récit d'une légende, d'une histoire le plus souvent liée au quotidien. Ces contes doivent toujours être dits le soir afin de ne pas porter malheur. Cet aspect est important car on ne peut s'empêcher d'établir un lien avec le personnage, qui, comme le conteur africain, discute à bâtons rompus le soir, non pas autour de l'arbre à palabres mais dans le "Paddy's Bar ", son équivalent urbain américain en quelque sorte. Autre point commun, le conteur africain est un acteur avant tout qui doit rendre son histoire vivante s'il veut être entendu et 
compris de tous ; il utilise l'humour dans ses mises en scène. Comme le conteur africain, Simple sait faire rire ou sourire tout en dédramatisant des situations graves ou sérieuses. Il se rapproche d'autant plus du conteur africain que ses qualités théâtrales, son sens du geste et du verbe sont indissociables de sa personnalité d'orateur : "When the Lord said, 'Let there be light,' and there was light, what I want to know is where was us colored people?» (Temptation, 25).

Le côté nostalgique de Simple et son évocation du passé nous font aussi voir en lui les qualités du griot car Simple reste le conteur de la grande histoire du monde :

"The words in my song would just say a black man saw a star and followed it till he came to a stable and put his presents down. But the music would say he also laid his heart down, too which would be my heart. It would be my song I would be making up (Christmas Song, 180).

Simple est tour à tour poète (A Toast to Harlem, 20-23; Wooing the Muse, 28-34) et conteur puisque ses histoires allégoriques amusent certes, mais elles demeurent avant tout des enseignements et suscitent une prise de conscience comme dans There Ought to be a Law (61-64), ou encore Two Sides Not Enough (213) : "Once an egg gets in the frying pan, it has only two sides, too. And if you burn the bottom side, it comes out just like the race problem, black and white, black and white." Simple est aussi griot lorsqu'il fait revivre le passé et devient le narrateur de l'histoire du monde (Income Tax, 64-69). Cependant, Simple est conteur avant tout car il a recours aux métaphores, aux figures allégoriques mais encore au double langage. Pour Deleuze, ce sont là des manifestations du «balbutiement » qui permettent de substituer à la langue et aux styles dominants d'autres expressions langagières et d'autres modulations écrites depuis la marge. Par ce précédé, Hughes peut rendre certaines des histoires de Simple hermétiques aux noninitiés :

By the times Congress convenes, I'll be without means. Besides, I don't get enough for my taxes. I wants to vote down South. It's hell to pay taxes when I can't even vote down home. Taxation without representation is tyranny, so the books say (Income Tax, 65).

21 Les « contes » à valeur didactique de Simple ont une fonction sociologique et politique. Comme dans les contes africains qui définissent la place de l'homme dans la société africaine, ses contes définissent la place du Noir dans la société américaine du début du vingtième siècle et oriente son action ${ }^{10}$ :

“Color, color, color!" I said. "It's so easy to blame all one's failures and difficulties on color. To whine I can't do this, I can't do that because I'm colored - which is one bad habit you have, friend, always bringing up race."

"I do", said Simple, "because that is what I am always coming face to face with race. I look in the mirror in the morning to shave - and what do I see? Me. From birth to death my face - which is my race - stares me in the face (Present for Joyce, 173).

Par conséquent, l'expérience forgée sur le sol américain fait de Simple ce conteur africain-américain des temps modernes, porte-parole des Noirs et de la condition noire du début du vingtième siècle : "What I want is a part-time job with full-time pay, or else a position where you take a vacation all summer and rest all winter. But I am colored, so I know nothing like that is going to happen to me " (Present for Joyce, 173). En définitive, Simple est à la fois poète, conteur, griot ou bouffon, car Hughes lui impose des changements de statuts pour mieux nous tromper quant à ses intentions réelles et entretenir la confusion. Ce style-patchwork, pour ainsi dire, est une 
succession d'emprunts à la tradition européenne, américaine et africaine que Hughes exploite et modifie à son gré afin de créer son propre style.

\section{L'écriture de l'oralité}

Pour ajouter à la confusion, Hughes introduit un autre effet stylistique pour accentuer le sentiment d'aliénation du lecteur. En effet, le langage de Simple est perverti par l'intrusion du vernaculaire, c'est-à-dire l'écriture de l'oralité. Comme l'analyse Isabelle Rouffineau dans son étude sur The Color Purple:

Cet effet de style, qui recherche une authenticité qui ne manque pas d'artificialité, introduit une confusion supplémentaire dans la communication. (...) Le lecteur est rendu doublement actif pour appréhender le sens (Rouffineau 127).

En s'éloignant de l'anglais standard, Hughes montre toute sa créativité mais aussi celle de la langue orale noire dont la musicalité rythme les monologues et les échanges de Simple. Cette technique témoigne non seulement de la richesse de la langue noire vernaculaire mais aussi de la réussite esthétique de Hughes, écrivain de l'oralité. Les échanges suivants illustrent notre propos :

I am tired of trickeration (Conversation on the Corner, 13).

I tell you, winter is a worriation (Letting off Steam, 36).

I am glad I am not as ageable as you (Shadow of the Blues, 166).

You know, white folks would not put up with Jim Crow, if they ever got Jim Crowed themselves (Duty Is Not Snooty, 198).

Whatever you are talking about with your see-antics [semantics], Jack, at my age a man gets tired of the same kind of eggs each and every day (Two Sides Not Enough, 214).

Cette création langagière se retrouve aussi dans la traduction française de The Best of Simple ${ }^{11}$ même si certaines traductions, dont le titre, ne mettent pas forcément en valeur la création de Hughes et ne retranscrivent pas les effets voulus par l'auteur. Par exemple, l'expression de Simple: «Jim Crowed» devient «jim-crowés» en français; «worriation» devient «des emmerdements». « Ageable» est traduit par: «Eh ben, je suis content de n'être pas aussi vieux que vous.» En revanche, l'expression «see-antics » que Simple utilise pour parler de sémantique (semantics) devient " sème antique ».

Dans la langue d'origine, outre l'effet comique, ces échanges révèlent la volonté de Hughes de maintenir son personnage dans l'oralité pour lui conférer toute son authenticité mais surtout pour créer une langue dans la langue, une pratique finalement propre à la « littérature mineure ». Hughes, par son processus de création langagière, s'affranchit de la norme en proposant une autre écriture. En sortant des canons littéraires et en les adaptant, Hughes poursuit un travail de déterritorialisation d'un système codé et normé pour le re-territorialiser puisqu'il le remodèle et l'adapte, caractéristiques propres à la littérature « mineure »:

«Majeur» et «mineur» ne qualifient pas deux langues, mais deux usages ou fonctions de la langue. (...) C'est une notion très complexe, celle de minorité, avec ses renvois musicaux, littéraires, linguistiques, mais aussi juridiques, politiques. Minorité et majorité ne s'opposent pas d'une manière seulement quantitative. (...) C'est pourquoi nous devons distinguer : le majoritaire comme système homogène et constant, les minorités comme sous-systèmes, et le minoritaire comme devenir potentiel et créé, créatif. (...) Il n'y a de devenir que minoritaire (Deleuze, Guattari MP 131-134). 
ghes entend, certes, créer ses effets mais il n'est pas sûr qu'il ait souhaité s'affirmer par rapport à une catégorie plutôt qu'à une autre. L'écrivain s'est certainement accommodé des contraintes de la culture dominante afin de proposer une écriture nouvelle au sein de la culture nationale, telle une écriture de la frontière qui s'est accommodée de ses marges. Aussi, dans ce travail de création langagière, les irrégularités grammaticales et syntaxiques, relayées par des effets typiques de prononciation, permettent à l'auteur de s'éloigner de l'anglais standard et ajoutent à l'effet caricatural créé et voulu par Hughes pour faire sourire tous ses lecteurs, blancs et noirs confondus :

"Here, I ain't scared to vote" (A Toast to Harlem, 21).

"To tell the truth, I has not heard of him at all" (Jazz, Jive, and Jam, 242).

"I do not know no place in the country where I am welcome" (Wooing the Muse, 31).

"Mens will have girl friends all over the world" (High Bed, 55).

Cette forme de langage (qui est plutôt bien rendues dans la version française ${ }^{12}$ ), donne l'occasion à l'auteur d'entretenir l'ambiguïté car en s'éloignant de l'anglais standard le lecteur pourra sourire de l'ignorance de Simple et penser que tous les Noirs s'expriment ainsi ; mais il est alors pris au piège de la condescendance. En effet, ces différences avec l'anglais standard seront perçues comme du « mauvais anglais », alors qu'elles sont pour Hughes une façon de montrer que le vernaculaire noir est une langue à part entière, avec sa propre grammaire et ses propres règles. Comme le souligne Geoffrey K. Pullum dans son article intitulé «African American Vernacular English is not Standard English with Mistakes », le vernaculaire noir est « une langue dans la langue » et ne doit pas être perçu comme le résultat d'erreurs grammaticales et syntaxiques :

\begin{abstract}
African American Vernacular English as a dialect of English deserves respect and acceptance differs strikingly from Standard English but there is no more reason for calling it bad Standard English than there is for dismissing Minnesota English as bad Virginia speech, or the reverse. (...) AAVE shows that it has certain regular syntactic principles of its own (...). Languages have many rules and regularities of sentence structure and speakers select from the possibilities in ways that are highly complex. That is a sign of having a sophisticated and flexible grasp of the possibilities in a rule system (Pullum 48).
\end{abstract}

Dans son travail d'écriture, l'originalité linguistique dont fait preuve Hugues à travers ses créations lexicales témoigne du double décalage entre la langue standard et celle de la communauté noire qui doit à la fois assumer son oppression et en même temps revendiquer son authenticité. Toutefois, il existe un autre niveau de décalage si l'on considère Simple et Joyce (sa petite amie) puisqu'ils sont, tous les deux, les parfaites illustrations de l'opposition au sein même de la communauté noire. Dans la nouvelle Jazz, Jive, and Jam (239-245) Simple fait sourire le lecteur et sert de faire-valoir à Joyce lorsqu'il lui demande :

“ 'Dr. Conboy is smart,' says Joyce. 'Did you hear him quoting Aristotle?'

“ 'Who were Harry Stottle?' I asked.

“ 'Some people are not even misread,' said Joyce. 'Aristotle was a Greek philosopher

like Socrates, a great man of ancient times.'

“' 'He must of been before Booker T. Washington then,' I said, 'because, to tell the truth, I has not heard of him at all' (Jazz, Jive, and Jam, 242).

Ce passage révèle en filigrane à quel point Simple et Joyce incarnent ce décalage. Un décalage reflété, ici, par un dispositif «lexico-grammatical» totalement créé par Hughes. En faisant utiliser une langue «dégradée » à son personnage, ni totalement 
«jive », ni totalement «negro dialect », Hughes montre à quel point il s'accommode stratégiquement de la langue standard pour donner toute son authenticité à son personnage dont le décalage langagier est à la fois sa marque identitaire et d'opprobre :

“' 'Jess, watch your grammar in public. Don't say is you, and you is. That is not proper,' Joyce says.

'I begs your pardon,' I says. 'Forgive my down-home talk.'

'Down-home or up North,' says Joyce, 'such terms is not correct. You know it. You have been to school.'

'Colored school,' I said. Which is neither here nor there as long as you understand what I am saying' (Shadow of the Blues, 172).

En réinvestissant les stéréotypes, Hughes se les réapproprie ce qui lui permet de remettre subtilement en cause le système dominant. En définitive, cette forme de culture populaire permet aussi au personnage de tenir les problèmes sociaux et raciaux qu'il évoque à distance tout en renvoyant à la communication orale que les esclaves durent mettre au point :

La dissémination des esclaves dans le Sud interdit les regroupements d'ethnies africaines et l'expression d'un dialecte commun. Des éléments d'anglais s'articulent dans le negro dialect, instrument de communication orale. L'acculturation fragmentaire se fait par la pratique chrétienne et les Écritures dont la transmission orale demeure le seul canal admis. L'apprentissage de la lecture et de l'écriture étant interdit aux esclaves, l'usage, tombé en désuétude, des langues maternelles africaines ne peut donc se substituer à cette carence de l'expression (Royot, et.al 297).

Comme les esclaves avant lui, Simple crée son propre langage. Il pratique le jeu des dozens (jeu des douzaines ${ }^{13}$ ) à sa façon, ce qu'il explique à Boyd dans Feet Live Their Own Life (1-3) et aime parler le jive, l'argot de Harlem. Ce discours « d'une population des univers clos " (Royot 308) est un langage coloré, illustration de l'imagination des Noirs puisqu'ils créèrent le jive en réponse au vocabulaire standard inadéquat. Le jive est une langue intermédiaire, codée, où les règles grammaticales sont inexistantes et qui permet aux Noirs de communiquer entre eux. Il était d'ailleurs relativement difficile aux non-initiés de s'approprier ce langage, d'où l'utilité du Hepster's Dictionary de Cab Calloway ${ }^{14}$, à l'origine du «jitterbug ${ }^{15}$ » américain (auquel Simple fait référence) et du mouvement «zazou» en France. De nombreux magazines tentèrent d'ailleurs d'expliquer ce qu'était le jive :

Le jive a une étymologie propre, des règles strictes, un lexique, constitué de près d'un millier de mots et d'expressions infinies, qui s'enrichit constamment. Burley dit du jive que c'est " une langue en mouvement », et affirme que vous ne pouvez comprendre cette langue si vous ne comprenez pas les personnes qui l'utilisent. Le jive est le réflexe de défense des Noirs (Anderson 316) ${ }^{16}$.

Le jive de Simple regorge d'expressions de sa création: «she jumps salty " (Landladies, 5), "I'm tired of trickeration " (Conversation on the Corner, 13), "to be jim-crowed" (Temptation, 27), « hysterian/historian », (An Auto-Obituary, 239) ${ }^{17}$. Toutes ces expressions sont autant d'exemples de ce mode d'expression propre aux Noirs de Harlem. Le jive va aussi de pair avec la gestuelle et les inflexions de voix, ce que nous imaginons très bien avec Simple. Si nous lisons ses échanges à haute voix, les mots de Simple acquièrent leur musicalité, ses descriptions sont toujours très rythmées, ce qui donne au langage tout son attrait et sa spontanéité. Simple est indissociable de ce mode d'expression haut en couleur qui lui apporte toute sa spécificité :

Toutes les erreurs d'élocution de Simple font partie de son intelligence, elles sont ainsi neutralisées, voire transformées. Simple donne non seulement toute sa dignité 
au dialecte, mais il arrive à rendre l'anglais standard peu adapté en comparaison (Levin 231) ${ }^{18}$. en entretenant la confusion. Ce mode d'expression se traduit d'ailleurs en français par «propos nègre-blanc, baratin ${ }^{19}$ ». Le jeu entre le dit et le non-dit, l'illusion et la réalité n'est donc jamais très éloigné, car s'il souhaite avant tout faire rire, Hughes ne manque aucune occasion de perdre son lecteur sur le chemin du double sens et du doubleentendre. Ce mode d'expression, empreint d'une bonne dose d'humour, donne son individualité à Simple qui dévoile ses états d'âme en toute sincérité. Dans Le plaisir du texte, Roland Barthes évoque le pouvoir du langage institué mais parle aussi de «redistribution» du langage, un compromis adopté par Hughes qui glisse constamment de la marge à la norme et de la norme à la marge et inscrit son texte dans la « littérature mineure » :

Comme dit la théorie du texte : la langue est redistribuée. Or cette redistribution se fait toujours par coupure. Deux bords sont tracés : un bord sage, conforme plagiaire (il s'agit de copier la langue dans son état canonique, tel qu'il a été fixé par l'école, le bon usage, la littérature, la culture), et un autre bord, mobile, vide (apte à prendre n'importe quels contours), qui n'est jamais que le lieu de son effet : là où s'entrevoit la mort du langage. Ces deux bords, le compromis qu'ils mettent en scène, sont nécessaires (Barthes 13). réinvestissant les différents canons littéraires, Hughes glisse bien de la marge à la norme en mettant en scène ce "compromis » dont parle Barthes. Cette "ligne de fuite » révèle à la fois l'opposition de l'auteur à la norme mais aussi son désir de la faire sienne pour plaire aussi bien au lectorat noir qu'au lectorat blanc. Le glissement qui s'opère ici est en lien avec le nom même du personnage principal. L'onomastique est donc incontournable car le nom du personnage fonctionne aussi comme un palimpseste, tout comme les histoires qui se superposent les unes aux autres à travers ce personnage « simple » et complexe à la fois.

travers le nom de Simple et ses multiples interprétations, Hughes adopte la « ligne de fuite » et se plaît à jouer sur le rapport signifiant/signifié pour conférer toute son ambiguité au texte. La stratégie du «naming» fait effectivement écho au texte de Hughes dans son ensemble. Le nom de ce protagoniste a été créé sur un jeu de mots. L'expression courante "Just be simple " (que nous retrouvons dans le nom même du personnage "Jess B. Semple») signifie: "fais pas l'idiot!», or cette apparente simplicité cache une multitude de significations et d'interprétations. Et si son entourage et ses proches considèrent Simple comme un doux rêveur voire un simple d'esprit, il se défend, en revanche, d'être stupide et naîf; ce qui est au contraire renforcé avec la traduction française puisque Simple est devenu L'ingénu de Harlem. Simple nous alerte donc sur les différents niveaux d'interprétation que nous pourrons donner à ses propos et à ses histoires :

" 'Joyce, after all these years, is you trying to play me for a sucker? If you is, lemme tell you - I might look simple, but I definitely ain't no fool"' (Once in a Wife-Time, 172).

Il attribue plutôt ce défaut à son aïeul :

" 'You have to ask my great-great-grandpa why. He must of been simple - else why did he let them capture him in Africa and sell him for a slave to breed my greatgrandpa on slavery to breed my grandpa in slavery to breed my pa to breed me to look at that window' "(Feet Live Their Own Life, 3). 
le simple » et que l'on continue toujours à l'appeler le « Simple d'esprit » :

“ 'I am really Jess Semple - which the kids changed around into a nickname when I were in school. In fact, they used to tease me when I were small, calling me 'Simple Simon'. But I was right handy with my fists, and after I beat the 'Simon' out of a few of them, they let me alone. But my friends still call me Simple"' (Simple on Indian Blood, 18-19).

Signalons aussi que "Semple » se prononce à la fois Simple et Semple. Ainsi, la première signification que nous retenons est celle du simple d'esprit, rêveur et optimiste que personne ne prend au sérieux et qui peut avouer sans crainte des vérités embarrassantes. Simple n'est finalement pas éloigné de la fonction du bouffon. En ce sens, il s'apparente au clown qui dénonce les maux de la société tout en faisant rire et donne à réfléchir sur la portée universelle de ses messages. Revêtant tantôt le costume du clown blanc, tantôt celui de l'Auguste, Simple envisage le monde sous diverses perspectives à la fois pessimistes et optimistes. Son imagination débordante l'aide à oublier les vicissitudes de la vie et sa condition (et par extension celle de son peuple). Comme il l'avoue dans High Bed (52-57) s'élever littéralement dans les airs lui permettrait de s'élever moralement et de transcender tout ce qui l'accable, ce qui fait dire à Boyd que son ami est bien « simple d'esprit » :

" 'Why, man, I would rock so far away from this color line in the U.S.A., till it wouldn't be funny. I might even build me a garage on Mars and a mansion on Venus. (...) Man, if I had a rocket plane, I would rock off into space and be solid gone. Gone. Real gone! I mean gone!'

"I think you are gone now," I said. "Out of your head."

"Not quite," said Simple (High Bed, 57).

Semple peut aussi être prononcé "sample ", c'est-à-dire " échantillon » et il est vrai qu'il n'existe pas meilleur représentant des habitants de Harlem et des Noirs; Simple incarne un archétype. En l'écoutant et en le regardant vivre et venir à bout de ses problèmes, ce sont ses semblables que nous voyons évoluer sous nos yeux dans le Harlem et l'Amérique des années 1920, ce que Hughes explique d'ailleurs en avantpropos :

I cannot truthfully state, as some novelists do at the beginnings of their books, that these stories are about "nobody living or dead." The facts are that these tales are about a great many people although they are stories about no specific persons as such. But it is impossible to live in Harlem and not know at least a hundred Simples, fifty Joyces, twenty-five Zaritas, a number of Boyds, and several Cousin Minnies or reasonable facsimilies thereof ${ }^{20}$.

41 Le prénom de Simple est également symbolique. Dans la bible, Jesse (Josué en français) est le père du roi David et il conduisit les Hébreux dans la conquête de la Terre Promise. Personne vertueuse, Josué enseignait la Torah aux foules. Dans The Best of Simple, Simple revêt le même rôle en dispensant un ensemble d'enseignements à travers son histoire et celle de la communauté noire. Ses prises de position sont une façon de faire prendre conscience à tous des problèmes inhérents aux Noirs et de donner une autre image d'eux. Or, Simple affirme dans Final Fear (57-61) qu'il aurait dû être prénommé Job et non Jesse car cet autre prénom aurait bien mieux convenu à sa situation : "Suffered!" Cried Simple. "My Mama should have named me Job instead of Jess Semple (Final Fear, 60). Effectivement, le prénom Job est particulièrement symbolique lorsqu'il renvoie à Simple. Homme de grande vertu et de piété, Job est mis à l'épreuve par Dieu et le Livre de Job raconte les circonstances de cette mise à l'épreuve. Si le lecteur peut douter de la 
grande vertu de Simple, il n'en reste pas moins que le rapprochement avec Job est fondamental. Lorsque Simple évoque sa condition et ses problèmes quotidiens, le lexique de l'injustice et des mauvais traitements, mais aussi l'accumulation de termes construits avec le préfixe « sous » sont un parallèle avec la punition divine de Job :

I have been underfed, underpaid, undernourished, and everything but undertaken. I been bit by dogs, cats, mice, rats, poll parrots, fleas, chiggers, bedbugs, granddaddies, mosquitoes, and a gold-toothed woman. (...) In this life I been abused, confused, misused, accused, false-arrested, tried, sentenced, paroled, blackjacked, beat, third-degreed and near about lynched!" (Final Fear, 60).

Tout comme Job, l'infortune de Simple est source d'expérience et d'enseignement non seulement pour lui mais aussi pour le lecteur. En évoquant l'origine de son nom, Simple se donne pour ambition de créer sa propre généalogie et de constituer son identité propre, ce qui fut refusé à ses ancêtres esclaves. Cette dénomination est pour Simple une façon de s'inscrire dans l'histoire commune nationale et de s'affranchir de son passé familial d'esclave. Pouvoir choisir son nom est un moyen, pour lui, d'affirmer son identité.

A la manière du bluesman qui raconte des micro-histoires Hughes fait parler l'ensemble des Noirs à travers son personnage. En faisant dire « je » à Simple, Hughes se met à la place de chaque Noir et abolit subtilement la frontière entre fiction et réalité tout en jouant avec son lecteur qui doit décoder les différents messages qui se tissent au fur et à mesure des échanges entre Simple et Boyd; échanges qui combinent d'ailleurs adroitement écriture et oralité. Boyd, le narrateur, a en effet un talent pour l'écriture et avoue aimer les mots, alors que Simple, en homme du Sud, est avant tout un orateur, un conteur d'histoires : «I can talk, but I can't write» (Income Tax, 69). Simple est le porte-parole de Hughes et gagne en authenticité, au point que la frontière entre le personnage et l'auteur-narrateur s'estompe, tout comme la limite entre l'illusion et la réalité.

Chaque histoire ajoute davantage à la confusion tout en créant des couches de sens successives. En définitive, Hughes propose un personnage qui lui permet de se faire entendre sans jamais se révéler directement; il s'aide d'une arme sans égal en la personne de Simple tout en jetant un regard rétrospectif sur la Renaissance de Harlem puisque ces histoires furent écrites bien après cette période faste. Nous avons donc là toutes les caractéristiques propres au personnage de Simple, amalgame de stéréotypes et portant différents masques pour faire passer son message. Hughes introduit dans son écriture autre chose que les stéréotypes auxquels les Blancs de son époque étaient habitués mais il s'appuie, en même temps, sur les idées reçues qui imprègnent la culture populaire américaine. Il associe ainsi les clichés des Blancs aux clichés que les Noirs ont d'eux-mêmes pour créer un personnage crédible et authentique, incarnation des contradictions de son temps.

\section{« Ligne de fuite » et « déterritorialisation »}

Le «langage du signifiant » permet d'appréhender la "double-voix» (double voice) inhérente aux textes des auteurs africains-américains où s'enchevêtrent des myriades d'histoires, de voix et de possibilités intertextuelles. La théorie du «signifying monkey " (singe signifiant) de Henry Louis Gates Jr. établit un lien entre les mythes africains (que les esclaves apportèrent, selon lui, avec eux dans le Nouveau Monde) et 
les œuvres littéraires des grands auteurs africains-américains ${ }^{21}$. Gates définit la figure $\mathrm{du}$ « singe signifiant » (signifying monkey), principe rhétorique du discours vernaculaire africain-américain, de la façon suivante :

Le «Singe Signifiant» est la figure de style par excellence du discours afroaméricain; dans la tradition afro-américaine, le langage du signifiant est sa forme verbale. (...) L'écriture est liée au mythe d'Esu, alors que l'oralité est propre au mythe du Singe Signifiant. (...) Dans la tradition, ces figures symbolisent la dualité de la voix. Comme de nombreux textes africains le prouvent, Esu et son ami le Singe sont eux-mêmes à la recherche d'une voix (Gates 22) ${ }^{22}$. africains-américains, car le va-et-vient entre le sens littéral et figuré peut être source de quiproquo pour un lecteur non averti. La théorie du « singe signifiant » s'appuie sur la figure du farceur ou du filou (trickster), un personnage mythique de la littérature africaine-américaine présent dans les différentes cultures africaines. Cette figure, indispensable au bon fonctionnement d'une société, détruit toute idée de permanence ou d'ordre social :

Trickster: personnage mythique qui joue un rôle consistant à dérégler le jeu normal des évènements, à plaisanter sur les dieux. Présent dans les cultures africaines, les missionnaires chrétiens l'ont souvent confondu avec le diable. ${ }^{23}$

Simple correspond bien à cette définition-là. Il est tour à tour sérieux et grave lorsqu'il défend la cause noire, et devient drôle et comique s'il évoque sa logeuse ou ses compagnons d'infortune. A cet égard, Simple porte la marque de son auteur tout autant que celle de son époque et est le produit de l'Histoire (la grande histoire) mais aussi de l'histoire qu'il met lui-même en scène. L'humour est ici le mode dominant qui permet à Hughes de «questionner les problèmes" de la société. Pour Hughes, l'humour est un moyen de réagir face à la réalité sociale d'une part, et de sonder l'esprit inhérent à la culture noire traditionnelle d'autre part. Construites comme de petites pièces de théâtre, les aventures de Simple sont une succession de saynètes qui jouent sur le comique de situation. Simple retrouve son interlocuteur principal, Boyd (le narrateur) et est entouré de personnages secondaires: Joyce, Zarita, sa logeuse, la logeuse de Joyce, les cousins F. D. et Minnie, Isabel, son ex-femme. Ces personnages, doublés de péripéties diverses, servent de source d'inspiration à Simple. En prenant le cadre du« Paddy's Bar» ou du "Wishing Well », l'illusion du réel est ainsi créée car ces histoires pourraient être racontées par quiconque dans un bar de Harlem.

Simple domine tous les sketches mais il gagne en efficacité grâce à la présence de Boyd qui, sans lui voler la vedette, donne plus de poids à ses prises de position. L'astuce de l'auteur consiste ainsi à manipuler le lecteur: les histoires commencent toujours par des propos inopportuns et inoffensifs pour mieux délivrer l'opinion de Simple in media res sur le quotidien, la question raciale, la violence, le racisme et les brimades dont les Noirs sont victimes. Ce jeu de palimpseste, voire de kaléidoscope, est infini puisque depuis le nom du personnage jusqu'à ses propos, ses histoires et ses tracas, les significations et interprétations se superposent et s'enchevêtrent et impliquent des significations, des «strates", plus ou moins évidentes pour le lecteur qui se doit de rester un lecteur actif. Aussi, nous pouvons interpréter l'affirmation suivante de Simple de deux façons différentes: «But when I opened the door, I hollered out loud, also damn near turned pale. I had not expected to see no Negro setting on my bed. I thought he were a robber (They Come and They go, 134) ». Pour un lecteur blanc ayant fait l'expérience de la ségrégation et favorable à la séparation raciale, les Noirs sont « tous 
des voleurs ». Pour Simple, utiliser les stéréotypes sans détour ou avec plus de subtilité est avant tout une façon de révéler comment la société américaine considère les Noirs et à quel point les stéréotypes raciaux ont la vie dure : "I don't do nobody no harm, do I? You don't see me out here hustling off nobody, do you? I am not mugging and cheating and robbing, I am?" (Conversation on the Corner, 13). Par ce moyen, Simple peut ainsi avouer sans crainte des vérités embarrassantes et dénoncer les maux de la société dans laquelle il vit. Cette technique de l'intertextualité suppose un degré d'implication du lecteur qui doit savoir lire entre les lignes, interpréter et analyser ce que lui livre le personnage, car ce mouvement de va-et-vient entre le littéral et le figuré suppose « une troisième oreille ", une forme de connivence entre le lecteur et son auteur en quelque sorte. Le lecteur tient sa propre carte entre les mains; il suit le chemin qu'il a choisi en fonction de son interprétation et de son rapport à l'écriture de Hughes. En étant ainsi bousculé, le lecteur fait aussi l'expérience de la ligne de fuite car la modification des règles le pousse hors du canon littéraire, hors de son territoire et bouleverse son usage habituel de la langue :

La ligne de fuite est une déterritorialisation. (...) Fuir, ce n'est pas du tout renoncer aux actions, rien de plus actif qu'une fuite. C'est le contraire de l'imaginaire. C'est aussi bien faire fuir, pas forcément les autres, mais faire fuir quelque chose, faire fuir un système comme on crève un tuyau... Fuir, c'est tracer une ligne, des lignes, toute une cartographie (Deleuze, DCP 47).

Les oppositions binaires récurrentes qui rythment le texte dans son intégralité font partie intégrante de l'innovation rhétorique de Hughes qui proposa d'en finir avec la marginalisation littéraire africaine-américaine en imposant un style nouveau qu'il voyait comme le socle d'une véritable tradition littéraire. L'itinéraire de Simple est semé de discordances qui s'organisent en thèmes conflictuels. Les dichotomies existantes dans la vie des Noirs américains prennent toutes leurs significations à travers les péripéties de Simple. Le contraste ville/campagne, haut/bas de l'île de Manhattan (uptown/downtown), culture rurale/culture urbaine, tradition africaine/ tradition américaine, apparence/réalité et personnage/narrateur sont autant d'oppositions qui engendrent l'ambiguïté mais qui symbolisent avant tout l'expérience noire aux États-Unis et supposent une forme de connivence entre l'auteur et son lecteur puisque le point de vue proposé est toujours double et souvent contradictoire. Simple est donc en déséquilibre permanent entre ce qu'il dit et ce qu'il est car il est à la fois un homme du Sud avec une culture rurale mais il s'est urbanisé en s'installant à Harlem. En ce sens, il analyse la question raciale en homme du Sud et prête aux problèmes du Sud une importance qui dépasse le cadre des États sudistes. Deux mondes, l'un étant l'antithèse de l'autre, sont décrits afin de mieux souligner les conditions de vie dans le Sud, la ségrégation, la violence et l'humiliation qui poussèrent tant de Noirs à quitter les États du Sud et à tenter leur chance dans les villes du Nord:

"Winter time in Harlem sure is a blip (...)".

"One could be rich and go to Florida."

"You couldn't give me Florida and all that Jim Crow on a silver platter!" (Letting off

Steam, 36)

Simple rejette le Sud pour sa violence et les lois Jim Crow mais il reste profondément attaché à ses racines. Les binarités sur lesquelles repose toute l'histoire de Simple montrent que la réalité est autre et qu'Harlem n'est finalement pas le paradis tant attendu comme l'explique Simple lui-même à sa cousine Minnie fraîchement arrivée de Virginie : 
Set down and I will tell you about Harlem, Minnie, so you will be clear in mind. In fact, I will tell you about the North. Down South you're swimming in a river that's running to the sea where you might drown but, at least, you're swimming with the current. Up North we are swimming the other way, against the current, trying to reach dry land. I been here twenty years Minnie, and I'm still in the water, if you get what I mean (Enter Cousin Minnie, 209).

Harlem ne tint pas ses promesses et ne put faire face aux vagues massives d'immigrants du Sud ${ }^{24}$ et malgré de nombreux efforts pour combattre la dégradation, le quartier devint peu à peu un ghetto :

Harlem, like many other urban Negro communities, underwent its most radical years of transformation and Negro settlement in the 1920's. Community efforts were simply overwhelmed by the magnitude of the population change that took place (...). This, and the planlessness that historically typified American urban growth, especially in New York City, permitted Harlem to become the horrible slum it remains today (Osofsky, 153-155).

Le schéma binaire est aussi omniprésent dans le texte de Hughes qui codifie la ségrégation raciale et spatiale entre les Noirs et les Blancs :

I am sorry white folks is scared to come to Harlem, but I am scared to go around some of them. Why, for instant, in my home town once before I came North to live, I was walking down the street when a white woman jumped out of her door and said, 'Boy, get away from here because I am scared of you.'

"I said, 'Why'?

"She said, 'Because you are black.'

“(...) I got more reasons to be scared of white folks than they have of me"( A Toast to Harlem, 22).

Plusieurs oppositions sont mises en abîme ici. La première interprétation qui se dégage de cet échange est bien sûr le constat de la ségrégation entre Blancs et Noirs. Nous savons que les Noirs furent confinés à Harlem et ainsi tenus à l'écart des Blancs. Les Blancs qui osèrent s'aventurer à Harlem à la nuit tombée n'eurent que très peu de contacts avec leurs habitants puisque les cabarets présentaient de grands artistes noirs sans pour autant autoriser l'accès au public noir. Comme dans le Sud, la ségrégation raciale s'accompagne de la ségrégation spatiale.

Les débats idéologiques et politiques des leaders noirs se retrouvent aussi dans les aventures de Simple. En aspirant à un renouveau, les intellectuels noirs ne tombèrent pas tous d'accord sur les principes à mettre en place et différents courants de pensée furent revendiqués. Contrairement à simple qui est favorable à un repli communautaire et préfère vivre avec ceux de sa race, Joyce prône l'intégration et les relations interraciales. Pour ce faire, le Noir qui véhicule une image négative, selon Joyce, se doit de modifier cette image afin d'être accepté :

“'Did you not hear him say that Negroes have played a part in all history, throughout all time, from Eden to now?

“' Do you reckon Eve was brownskin?' I requested.

“" I do not know about Eve,' said Joyce, 'but Cleopatra was of the colored race, and the Bible says Sheba, beloved of Solomon, was black but comely. Solomon also found Cleopatra comely. He was a king,' says Joyce (Jazz, Jive, and Jam, 242).

55 A cet égard, la culture est, pour elle, une solution essentielle. Comme le leader noir Booker T. Washington (1856-1915), Joyce croit au développement de l'éducation, unique facteur d'émancipation, un point de vue que Simple ne partage pas totalement. En donnant à Joyce et à Simple l'occasion d'exprimer différents points de vue, Hughes met en scène le débat idéologique qui opposa deux grands leaders noirs du début du 
vingtième siècle: Booker $\mathrm{T}$. Washington et William E. B. Du Bois. Récusant le gradualisme de Washington qui recommanda d'accepter la ségrégation tout en renonçant à l'agitation politique, Du Bois milita pour les droits civiques des Noirs en revendiquant leur dignité. Par conséquent, Simple et Joyce sont l'expression de ce débat et de ces tensions idéologiques au sein de la communauté noire. Ils donnent à envisager un modus vivendi qu'aurait pu adopter la communauté noire à cette époque où la solution aurait été à mi-chemin entre intégration et préservation de l'authenticité noire. Simple ne souhaite pas décevoir Joyce, mais il revendique ses origines avant tout et est prêt à en payer le prix, il reste un « nègre » et refuse finalement le compromis :

“I am race conscious," said Simple. “And I ain't ashamed of my race. I ain't like that woman that bought a watermelon and had it wrapped before she carried it out of the store. I am what I am. And what I say is: 'If you're corn bread, don't try to be an angel-food cake! (Midsummer Madness, 126).

Cette différence entre les personnages principaux traduit finalement les contradictions de la Renaissance de Harlem et l'expérience noire aux États-Unis dans sa globalité. Cependant, en opposant Simple et Joyce l'auteur réussit à créer une connivence entre lui et ses lecteurs puisqu'il propose deux types d'idéaux, deux attitudes, qui engendrent certes la confusion mais supposent que chacun y trouve son compte. La position ambiguë de Simple provient à la fois de son mode de communication et de sa fonction documentaire de la condition noire dans une grande ville du Nord au début du vingtième siècle. Hughes, en adepte du double-entendre, utilise le masque de Simple pour évoquer de façon indirecte et subtile le racisme, la ségrégation, la discrimination et présenter de manière efficace et lucide les multiples problèmes qui se posèrent avec acuité aux Noirs de son époque.

Les composantes qui définissent la « littérature mineure » selon Deleuze et Guattari ainsi que les opérations de minoration qui la constitue sont bien présentes dans l'écriture de Hughes et dans The Best of Simple. Hughes utilise le vernaculaire noir américain comme un moyen de minorer l'anglais standard et utilise un amalgame de genres littéraires pour mieux sortir du canon littéraire et créer sa propre littérature. Par ce procédé Hughes refusa l'académisme artificiel et inadapté à son processus de création. D'autres auteurs avant lui eurent recours à l'utilisation des dialectes dans leur écriture et menèrent aussi une réflexion sur ce thème. Toutefois, Langston Hughes va plus loin et offre au lecteur une création esthétique originale sur le mode d'une oralité réminiscente du blues et du jazz.

En faisant parler le jive et la langue noire vernaculaire à son personnage, Hughes minore la langue dominante et montre son refus d'un académisme formel et artificiel. Par ce procédé de déterritorialisation, Hughes réussit à reterritorialiser un mode d'expression à la fois dans sa communauté et en dehors. Navigant sans cesse entre la norme et la marge, Hughes donna naissance à une écriture métissée et musicale, une écriture qui ne trouve nul équivalent dans la littérature américaine. «Les concepts de modulation et de minoration donnent alors à penser une immanence réelle des normes » (Sibertin-Blanc) car en créant une nouvelle écriture, Hughes crée de nouvelles règles, de nouvelles normes en étendant ses variables jusqu'à leurs limites.

Peu connu en France, The Best of Simple (ou L'ingénu de Harlem) est entré dans la tradition littéraire noire américaine car Hughes sut plaire à la fois au lectorat blanc et noir à travers le personnage de Simple. Par sa force de conviction et sa valeur critique de la culture dominante, mais aussi par son traitement de l'héritage littéraire africain- 
américain Hughes laissa une autre empreinte et amorça un nouveau tournant dans la littérature africaine-américaine.

\section{BIBLIOGRAPHIE}

Anderson, Jervis. This Was Harlem. A Cultural Portrait, 1900-1950. New York: The Noon Press, 1981.

Barthes, Roland. Le plaisir du texte. Paris: Editions du Seuil, 1973.

Ira, Berlin. The Making of African America. The Four Great Migrations. New York, Penguin Books, 2010.

Deleuze, Gilles et Félix Guattari. Mille Plateaux. Paris: les Editions de Minuit, 1980.

Deleuze, Gilles. Critique et clinique. Paris: Les Editions de Minuit, 1993.

---, Dialogues, avec Claire Parnet. Paris: Flammarion, 1977.

---, Kafka, pour une littérature mineure. Paris: Les Editions de Minuit (collection « Critique »), 1975.

Fabre, Michel. « L'Amérique blanche à la découverte de son âme noire ». In, Isabelle Richet (dir.), Harlem 1900-1935, Paris, Éditions Autrement, Série Mémoires - n² 25, 1993.

Gates, Henry Louis Jr. The Signifying Monkey. A Theory of African-American Literary Criticism. New York and Oxford: Oxford University Press, 1988.

Hecquet, Vincent. «Littératures orales africaines », Cahiers d'études africaines [En ligne], 195 | 2009, 833-840, mis en ligne le 22 septembre 2009, consulté le 08 juillet 2015. URL : http:// etudesafricaines.revues.org/14052

Hughes, Langston. The Best of Simple. New York : Hill \& Wang, 1961. Traduit par F. J. Roy, L'ingénu de Harlem. Paris : La Découverte, 2003.

Levin, Harry. Veins of Humor. Cambridge, Massachusetts: Harvard University Press, 1972.

Osofsky, Gilbert, Harlem: the Making of a Ghetto. New York: Harper Torchbooks, second edition, 1971.

Pullum, Geoffrey K. «African American Vernacular English is not Standard English with Mistakes », in Rebecca Wheeler (ed.), The Workings of Language. Westport, Connecticut: Praeger, 1999.

Richet, Isabelle (dir.). Harlem 1900-1935, Paris : Éditions Autrement, Série Mémoires - nº 25, 1993.

Rouffineau, Isabelle. The Color Purple ou la Féminitude Pourpre. In, La Couleur du Temps dans la culture afro-américaine. Textes réunis par Claudine Raynaud, Actes de l'Atelier de l'AFEA du 22-23 mai 2004. Cahiers de Recherches Afro-Américaines Transversalité $n^{\circ} 1 / 2005$. Tours : Presses Universitaires François Rabelais, 2005.

Royot, Daniel, et al. Histoire de la culture américaine. Paris : Presses Universitaires de France, 1993. Sibertin-Blanc, Guillaume. «Pour une littérature mineure : un cas d'analyse pour une théorie des normes chez Deleuze ». [En ligne] Université de Lille 3, 2003. Disponible sur 
http://stl.recherche.univlille3.fr/seminaires/philosophie/macherey/Macherey20022003/

Sibertn.html (consulté le 27 avril 2015).

\section{NOTES}

1. Arnold Rampersad, biographe de Langston Hughes et critique littéraire, utilise l'expression «mulatto-like text » (que je traduis par « texte métissé») pour décrire la poésie de Hughes. Cette expression est particulièrement adéquate pour décrire le travail de Hughes qui souscrit à certaines conventions et emprunte à la fois à divers genres littéraires classiques et au folklore africain-américain dans son écriture.

Voir l'article: Arnold Rampersad, «Hughes's Fine Clothes to the Jew », in K. A. Appiah et H. L. Gates Jr. (éd.), Langston Hughes, Critical Perspectives Past and Present. New York, Amistad, 1993, p. 58. 2. Ces nouvelles furent réunies en 1961 sous le titre The Best of Simple. La traduction française par F. J. Roy est intitulée, L'ingénu de Harlem. La version originale sera utilisée pour cet article.

3. J'emprunte ce terme à Roland Barthes qui, dans Le plaisir du texte, dénonce le pouvoir du langage institué. Langage que Hughes déconstruit et reconstruit à loisir dans son œuvre et dans l'ouvrage analysé ici.

4. Deleuze nomme "rhizome» un système fait de connexions et d'hétérogénéité : "n'importe quel point d'un rhizome peut être connecté avec n'importe quel autre et doit l'être. (...) Un rhizome peut être rompu, brisé en un endroit quelconque, il reprend suivant telle ou telle de ses lignes en suivant d'autres lignes. (...) La ligne de fuite fait partie du rhizome. Ces lignes ne cessent de se renvoyer les unes aux autres » (Deleuze, Guattari, MP, pp. 13 et 16).

5. Déterritorialiser est envisageable, pour Deleuze, avec son pendant : la reterritorialisation. Se déterritorialiser signifie quitter la norme et s'affranchir de la norme pour mieux échapper au modèle dominant et se rapprocher du « devenir-révolutionnaire ».

6. Les erreurs de syntaxe sont un choix délibéré de l'auteur pour son personnage et participe de cette création caractéristique de la « littérature mineure ».

7. Walden ou la vie dans les bois (Walden or Life in the Woods) fut publié en 1854 par Henry David Thoreau (1817-1862) et fait partie des ouvrages phares de la littérature américaine. Le livre raconte la vie de Thoreau dans les bois où il resta pendant deux ans. Ce livre est une critique d'un monde marqué par l'industrialisation où l'homme perd conscience de son environnement. Walden est le roman du retour à la nature.

8. Ben Jonson (1572-1637), dramaturge anglais connu pour ses comédies de caractère : Volpone (1606) et L'alchimiste (1610). Contemporain de Shakespeare, sa réputation égala celle de ce dernier.

9. Dans son article intitulé «Littératures orales africaines », Vincent Hecquet constate : "Dans la société igbo du Nigeria, chaque genre est pratiqué de façon privilégiée par un groupe social. Les hommes investissent dans les discours politiques et les proverbes, registres les mieux considérés qui permettent d'exprimer des talents d'orateur et de concentrer une sagesse ancestrale. Le conte, considéré comme une parole moins sérieuse, est avant tout l'apanage des femmes. Les enfants pratiquent la devinette, la comptine et apprennent à raconter des contes. " Hecquet renvoie aussi à : Baumgardt, Ursula \& Ugochukwu, Françoise, Approches littéraires de l'oralité africaine. Paris : Karthala, 2005.

10. Le conte africain s'inscrit dans le contexte culturel d'une communauté. Selon Vincent Hecquet : « comme les épopées orales, les contes reflètent les structures et valeurs sociales. Les Contes recueillis et analysés par Christiane Seydou illustrent les institutions peules traditionnelles : monarchie, hiérarchie sociale, polygamie, pastoralisme » (Hecquet, 837).

11. Il s'agit de L'ingénu de Harlem. Traduction de F. J. Roy. 
12. Par exemple, on trouve des expressions telles que : "C'est moi qui a la parole » (Simple et le sang indien, 38); «Joyce, je lui dis, j'ai pas envie de faire aucune photo» (Une photo pour son dressoir, 157) ; "Je suis couru aller me chercher le permis » (L'amour tout simplement, 277) ; « Où c'est qu'elle va, je vas » (L'amour tout simplement, 279). « De nos jours, si quelqu'un tomberait sans connaissance dans un enterrement, l'infirmière lui colle si tellement des sels sous le nez qu'il se met à éternuer » (Un sacré enterrement, 280). « Si je serais au Mississippi » (Radio-activité, 303).

13. Ce jeu de devinettes et d'insultes verbales est une façon ludique et orale de se protéger de l'agressivité des Blancs et des relations raciales.

14. Cab Calloway (1907-1994): chef d'orchestre et chanteur de jazz très populaire, ses onomatopées et vocalises avec l'orchestre, notamment son célèbre $\mathrm{Hi}$ de $\mathrm{Hi}$ de Ho, firent sa renommée.

15. À la fin des années 1930, le terme «jitterbug » désignait à Harlem une danse très populaire mais aussi les danseurs et toutes personnes dont la façon de s'exprimer et de s'habiller était excentrique. Comme Cab Calloway, les «jitterbugs » de Harlem adoptèrent le «zoot suit », un costume trois pièces composé d'une très longue veste, très épaulée, et porté avec des chaussures à bouts pointus et un chapeau; il n'y avait aucune limite imposée dans le choix des couleurs. Ces personnes étaient appelées «hep cats» (zazous). Lors d'une tournée en France, Cab Calloway inspira le mouvement zazou et ses onomatopées furent reprises en 1938 par Johnny Hess dans Je suis swing.

16. Ma traduction. Anderson cite le magazine Esquire de 1944.

17. Le lecteur français retrouve la création langagière de Hughes à travers certaines expressions du traducteur: "se mettre à sinuer (insinuer)» (Jalousie, 68), "être culturé » (Sept coups de sonnette, 145), "se précautionner » (Sur un lit d'hôpital, 83), «être scandaleur » (Rien qu'une fois dans la vie d'une femme, 250).

18. Ma traduction.

19. Traduction proposée par le Larousse en ligne : http://www.larousse.fr/dictionnaires/anglaisfrancais/jive [Site consulté le 2 mai 2015].

20. Langston Hughes, « Foreword: Who is Simple? », The Best of Simple, op. cit., p. vii.

21. Depuis sa publication, la théorie de Gates a été largement critiquée pour n'accorder aucune place au modernisme et au lyrisme dans l'écriture noire américaine. Elle apporte cependant un éclairage au texte de Hughes. Voir notamment l'article de Joyce A. Joyce, “A Tinker's Damn: Henry Louis Gates, Jr., and The Signifying Monkey Twenty Years Later". Callaloo, vol. 31, 2, Spring 2008, pp. 370-380.

22. Ma traduction.

23. Définition extraite du dictionnaire Larousse en ligne: www.larousse.fr/dictionnaires/ francais/trickster [Site consulté le 2 mai 2015].

24. A ce propos, Ira Berlin constate d'ailleurs: If greatness is measured by size, the Great Migration was great indeed. Between America's entry into the European war and the stock market crash in 1929, black men and women left the South at an average rate of 500 per day, or more than 15,000 per month. The evacuation of the black belt was particularly striking. (...) By 1930, more than 1.3 million resided outside the South, nearly triple the number at the turn of the century (Berlin, 154). 


\section{RÉSUMÉS}

Après avoir exploré différentes formes poétiques dans son écriture, Hughes créa Simple, un personnage fictif de nouvelles écrites sur une période de vingt-trois ans, qui devint le personnage phare de son œuvre. Ce personnage cristallise les différentes «modulations » et «minorations» de l'écriture de Hughes au sens deleuzien du terme.

Dans cet article, je propose d'analyser l'écriture de Langston Hughes dans son recueil de nouvelles intitulé The Best of Simple en m'appuyant sur les concepts théorisés par Gilles Deleuze et Félix Guattari dans Mille Plateaux (1980) notamment. A travers ces concepts, nous verrons comment Hughes, en écrivant depuis la marge et en refusant le modèle linguistique dominant, réussit à glisser du mode majeur au mode mineur.

After exploring different poetic and aesthetic forms, Hughes created Simple, his famous character whose adventures were published under the title The Best of Simple. These stories are typical of the process of "modulations" and "minor literature" (minoration) as exposed by Gilles Deleuze and Félix Guattari in Mille Plateaux especially.

In this article, my intention is to look at Hughes's mode of expression in The Best of Simple using Gilles Deleuze and Félix Guattari's work and theory to show how Hughes, by writing from the margins, evaded the dominant linguistic models and thus moved from "major" to "minor literature".

\section{INDEX}

Keywords : musical writing, minor literature, deterritorialisation

Mots-clés : écriture musicale, littérature mineure, déterritorialisation

\section{AUTEURS}

\section{CHRISTINE DUALÉ}

Maître de conférences

Université Toulouse 1 Capitole.

CAS, Université Toulouse Jean Jaurès

christine.duale@ut-capitole.fr 